\title{
Physiological Profiles of Saudi Elite Judo Athletes
}

\section{Mohammad Ali Alahmadi}

${ }^{1}$ Department Physical Education and Sport Sciences, College of Education, Taibah University, Madinah, Saudi Arabia

\begin{abstract}
:
One of the most highly physiologically demanding combat sports is judo, which requires an excellent level of physical fitness to be successful in international competitions. Although the physical and physiological characteristics of high-ranked judo athletes are well established, the characteristics of Saudi judo athletes have not been determined. Therefore, the aim of this study was to assess the physiological profiles of Saudi elite judo athletes. Fourteen judo athletes (mean \pm standard deviation (SD) for age, $17 \pm 4$ years; body mass, $66.7 \pm 21 \mathrm{~kg}$; height, $160.2 \pm 13.6 \mathrm{~cm}$ ) performed a special judo fitness test (SJFT). Body fat percentage (BF\%) was determined using the bioelectrical impedance scale. Handgrip strength was measured using a handgrip dynamometer, and flexibility was determined by using a sit-andreach test. The mean \pm SD of the index of the SJFT was $16.2 \pm 1.6$. The means \pm SD of $\mathrm{BF} \%$, handgrip strength, and flexibility were $24.1 \pm 7.2(\%), 31.4 \pm 11.5(\mathrm{~kg})$, and $34.6 \pm 6.7(\mathrm{~cm})$, respectively. We concluded that the SJFT performance and handgrip strength were lower, and BF\% was higher in Saudi judo athletes compared to international elite judo athletes.
\end{abstract}

Keywords: Physical fitness, cardiovascular fitness, aerobic fitness, judo sport, combat sports

\section{Introduction}

One of the most highly physiologically demanding combat sports is judo, which requires an excellent level of physical fitness for success international competitions (Franchini et al. 2011a). It is well known that understanding the characteristics of elite athletes can provide insightful to determine what is required for competitive success (Agostinho et al. 2018; Callister et al. 1991; Franchini et al. 2011a; Franchini et al. 2007). The physical and physiological characteristics of high ranked judo athletes are well established (Franchini et al. 2011c; Torres-Luque et al. 2016). For example, physical fitness levels of judo athletes are evaluated using a special judo fitness test (SJFT). SJFT is the most common test used to evaluate judo athletes, allowing coaches to classify their athletes according to a classification levels developed for both male and female judo athletes (Franchini et al. 2011b; Franchini et al. 2009; Franchini, Takito and Bertuzzi 2005; Sterkowicz-Przybycień and Fukuda 2014). Physical fitness is related to SJFT performance (Lopes-Silva et al. 2018). Thus, highly ranked Judo athletes must achieve an excellent SJFT index, where the lower the index level the better the physical fitness level. For example, judo elite athletes typically have a low SJFT index, which is a positive combination of aerobic and anaerobic contributions (Franchini et al. 2017). Strength is also an important component of physical fitness and has been found to be critical in Judo sport, determining the results of judo competitions (Ache Dias et al. 2011; Blais and Trilles 2006; Franchini, 
Schwartz and Takito 2018; Franchini, Schwartz and Takito 2020; Kons et al. 2018). Flexibility is also a crucial component of physical fitness in Judo competitions because of the great neuromuscular demand involved in this type of combat sport (Fukuda et al. 2011; Sertić, Sterkowicz and Vuleta 2009). Moreover, body composition is regarded as a key element for competing at an international level in the judo sport. Therefore, judo athletes are recommended to obtain a target range of body fat percentage values to maximize their physiological capacity and performance (Franchini et al. 2011a; Franchini, Takito and Bertuzzi 2005).

However, although the physical and physiological characteristics of highly ranked judo athletes are well established, the characteristics of Saudi judo athletes have not been determined. Therefore, the aim of this study was to assess the physiological profiles of Saudi elite judo athletes.

\section{Methods}

Fourteen Saudi male judo athletes (mean \pm standard deviation (SD) for age, $17 \pm 4$ years; body mass, $66.7 \pm 21 \mathrm{~kg}$; height, $160.2 \pm 13.6 \mathrm{~cm}$ ) participated in this study. The study protocol and procedures conformed to International Ethical Guidelines. All participants signed an informed consent form.

\section{Anthropometry and Body Composition}

Body weight was measured to the nearest $100 \mathrm{~g}$ using a portable digital scale (Seca, Germany), and height was measured to the nearest $0.1 \mathrm{~cm}$ using a portable stadiometer (Seca, Germany). Body mass index (BMI) weight/stature2) was calculated from height and weight measurements.

Body fat percentage was measured without shoes in light indoor clothes using a bioimpedance analyser (OMRON, BF511 body composition monitor). According to the manufacturer's instruction manual with the participants in light clothes and without shoes, the bioelectric impedance measurement was performed by placing the feet together on foot electrodes with the weight equally distributed. The participant's knees and back were in a straightened position with their arms extended forward and holding the grip electrodes for a few seconds. The fat percentage reading appeared on the display unit and was then recorded.

\section{SJFT}

SJFT has been well described by Franchini et al. (1998) and Artioli et al. (2005). Briefly, three athletes in the same weight class and of similar heights are needed to perform the SJFT, which is composed of three periods (A, $15 \mathrm{~s}$; B and C, $30 \mathrm{~s}$ ) separated by 10 -s recovery intervals. The athlete begins the test in a position between two partners who are standing three meters away from each other. During each period, the athlete being evaluated throws the two partners as many times as possible using the "ippon-seoi-nage" technique. The athlete must complete as many throws as possible within the tested time. The heart rate was measured immediately after the end of the test and was measured again $1 \mathrm{~min}$ after completion of the test. A heart rate monitor (Polar RS400) was used to measure the heart rate. Then, the SJFT index was calculated as follows:

Index $=($ Heart rate after + Heart rate $1 \mathrm{~min}$ after $) /$ total number of throws.

Of note, the lower the index, the better the result.

\section{Handgrip strength}

Maximal isometric handgrip strength was measured using a handgrip dynamometer (Takei Kiki Kogyo ${ }^{\circledR}$ dynamometer) adjusted to the hand size. A single measurement was taken for each hand to obtain the maximal isometric handgrip strength value. Each subject was instructed to maintain maximal isometric contraction during each 
measurement for 3 to 6 s (Kamimura and Ikuta 2001; Trossman and Li 1989). Contractions were made using each hand with both feet on the floor, the shoulder bent by $90^{\circ}$, and the elbow completely extended.

\section{Flexibility}

Flexibility was determined by using the sit-and-reach test. In this test, athletes sat with knees straight, feet flat on the bench, and both hands on top of each other with palms facing downwards. Then, each participant gradually inclined his trunk forward as far as possible. The athletes were not allowed to bounce or lunge. Athletes reached out and held that position for $2 \mathrm{~s}$, and then, the distance was recorded in centimetres $(\mathrm{cm})$.

\section{Statistical analysis}

Data were analysed by using the statistical software package SPSS, version 21. Descriptive statistics was presented as mean values with SD.

\section{Results}

Descriptive characteristics and physiological responses for all participants are shown in Table 1. The mean $( \pm$ SD) for the SJFT index was $16.2 \pm 1.6$. Of note, the lower the value for this component, the higher the elite judo athlete's performance.

Table 1 Physiological profiles of Saudi judo athletes

\begin{tabular}{|c|c|}
\hline Variables & Means ( \pm SD) \\
\hline Number of throws & $21.5 \pm 2.1$ \\
\hline Heart rate after the test (beats/min) & $186 \pm 12$ \\
\hline Heart rate 1 min after the test (beats/min) & $161 \pm 17$ \\
\hline SJFT index & $16.2 \pm 1.6$ \\
\hline Body fat percentage (\%) & $24.1 \pm 7.2$ \\
\hline Right handgrip strength (kg) & $31.4 \pm 11.5$ \\
\hline Left handgrip strength (kg) & $30.5 \pm 12.0$ \\
\hline Flexibility (cm) & $34.6 \pm 6.7$ \\
\hline
\end{tabular}

\pm SD: standard deviation; STJF: special judo fitness test; kg: kilogram; cm: centimetre

\section{Discussion}

To our knowledge, this is the first study to use the SJFT test to evaluate judo athletes in Saudi Arabia. The SJFT is the most common test used to evaluate judo athletes, allowing coaches to classify their athletes according to classification levels developed for both male and female judo athletes (Franchini et al. 2011b; Franchini et al. 2009; Franchini, Takito and Bertuzzi 2005; Sterkowicz-Przybycień and Fukuda 2014). The smaller the index value, the better the test performance. The current study showed that the mean $( \pm$ SD) SJFT index for the tested Saudi judo athletes was $16.2 \pm 1.6$, and when, we compared this SJFT value to international elite judo athletes, our determined SJFT index value was higher (SJFT = 12.7-13.2) (Casals et al. 2017). The lower performance in the SJFT in relation to international elite judo athletes is probably related to the lower aerobic power capacity of the tested Saudi judo athletes. The higher maximal oxygen consumption performance $\left(\mathrm{VO}_{2 \max }\right)$ found in judo athletes (Agostinho et al. 2018; Sterkowicz-Przybycień, Miarka and Fukuda 2016) may be an important factor contributing to the higher performance in the SJFT. It has been found that $\mathrm{VO}_{2 \max }$ is related to both an increased aerobic contribution to sprintrecovery bouts and the enhanced ability to resist fatigue during intense intermittent exercise (Tomlin and Wenger 2002). Most judo athletes have $\mathrm{VO}_{2 \max }$ values between 44 and $65\left(\mathrm{ml} / \mathrm{kg} / \mathrm{min}^{-1}\right)$, regardless of the protocols and exercise modes (Franchini et 
al. 2011a; Franchini et al. 2007; Nikolova, Dimitrova and Mladenov 2020; TorresLuque et al. 2016). A further study is required to investigate the relationship between SJFT index and aerobic fitness and to explain why SJFT index found in the current study was poor. Moreover, poor index SJFT of our judo athletes may suggests that Judo as combat sport seems to be anaerobic in nature rather than aerobic. Therefore, it is recommended for future research to investigate the relationship between anaerobic fitness such as Wingate test with SJFT. This relationship may explain partially the poor SJFT index found in the current study.

The average body fat percentage for the Saudi judo athletes in this study was higher (24.1\%) than those of international judo athletes (range of approximately $7 \%$ to $14 \%$ ) (Franchini et al. 2011a; Franchini, Takito and Bertuzzi 2005). Of note, body fat percentage may differ between heavyweight and non-heavyweight judo athletes. A recent Japanese study found that heavyweight judo athletes had a higher body fat percentage $(23.2 \%)$ compared to non-heavyweight judo athletes (13.0\%) (Murata et al. 2016). Body fat percentage has been found to negatively influence the physiological capacity and performance in judo athletes (Franchini, Takito and Bertuzzi 2005). For instance, body fat percentage was negatively correlated with the number of throws in the SJFT $(r=-0.69)$ (Katralli and Goudar 2012). Moreover, the body fat percentage for higher ranked elite judo players compared with lower ranked elite judo players differed significantly (Callister et al. 1991). Therefore, to maximize the physiological capacities and performances of Saudi judo athletes, a target range of body fat percentage should be obtained.

Additionally, handgrip strength is critical in judo and may determine the result of judo bouts because the gripping method (kumi-kata) is the first contact between two judo players, (Ache Dias et al. 2011; Blais and Trilles 2006; Kons et al. 2018). Fatigue in the upper limbs, especially in the hands, is common in judo. In fact, an overall decrease in the maximal isometric strength has been found during judo bouts because of fatigue in each hand (Bonitch-Góngora et al. 2011). Handgrip strength was not found to differ between judo athletes and non-judo athletes in absolute terms (peak grip force); however, judo athletes have been found to be more resistant to fatigue (Ache Dias et al. 2011). Generally, no difference has been found between elite and non-elite judo athletes (Franchini, Takito and Bertuzzi 2005). Our study showed that the handgrip strength of these Saudi judo athletes was $31.4 \mathrm{~kg}$, which is lower than that of international judo athletes for handgrip strength (ranges from 38 to $65 \mathrm{~kg}$ ) (Franchini et al. 2011a; Franchini, Schwartz and Takito 2018; Franchini, Schwartz and Takito 2020).

In addition to handgrip strength, flexibility is a crucial component of physical fitness in judo competitions because of the high neuromuscular demand involved in this type of combat sport (Fukuda et al. 2011; Sertić, Sterkowicz and Vuleta 2009). It has been shown that there is an inverse relationship between flexibility and SJFT index, meaning that the higher the flexibility, the better the performance in SJFT (Arazi, Noori and Izadi 2017). Although insufficient information exists regarding the flexibility of judo athletes, the flexibility the Saudi judo athletes determined in this study $(35 \mathrm{~cm})$ was similar to those reported by Marques et al. (2019). However, Arazi et al. (2017) found greater values for the flexibility in their judo athletes $(48 \mathrm{~cm})$ than of those in our study. 
To conclude, the SJFT performance and handgrip strength were lower and the BF\% was higher in Saudi judo athletes compared to international elite judo athletes. In the future, we would like to investigate the relationship between aerobic and anaerobic fitness with SJFT. This relationship could explain why the SJFT index found in this study was poor for these Saudi judo athletes compared to their peers. Thus, the results of this study suggest the need for more studies to investigate the influence of aerobic and anaerobic fitness variables on the judo-related performance in Saudi judo athletes.

\section{References:}

1- Ache Dias, Jonathan, M. Wentz, Wladymir Külkamp, Daniela Mattos, Márcio Goethel, and N. Júnior (2011): Is the handgrip strength performance better in judokas than in non-judokas?. Science \& Sports 27:e9-e14.

2- Agostinho, M. F., J. A. O. Junior, N. Stankovic, R. Escobar-Molina, and E. Franchini (2018): Comparison of special judo fitness test and dynamic and isometric judo chin-up tests' performance and classificatory tables' development for cadet and junior athletes. J Exerc Rehabil 14(2):244-52.

3- Arazi, Hamid, Mohsen Noori, and Mani Izadi (2017): Correlation of anthropometric and bio-motor attributes with Special Judo Fitness Test in senior male judokas. Ido Movement for Culture 17:19-24.

4- Artioli, Guilherme, Desiré Coelho, Fabiana Benatti, Alessandra Gailey, Patrícia Berbel, Thomás Adolpho, and Antonio Lancha (2005): Relationship Between Blood Lactate And Performance In A Specific Judo Test. Medicine \& Science in Sports \& Exercise 37:S99.

4- Blais, Laurent, and Francis Trilles (2006): The progress achieved by judokas after strength training with a judo-specific machine. Journal of sports science \& medicine 5(CSSI):132-35.

5- Bonitch-Góngora, Juan, Juan Bonitch-Domínguez, Paulino Padial, and Belen Feriche (2011): The Effect of Lactate Concentration on the Handgrip Strength During Judo Bouts. Journal of strength and conditioning research / National Strength \& Conditioning Association 26:1863-71.

6- Callister, R., R. J. Callister, R. S. Staron, S. J. Fleck, P. Tesch, and G. A. Dudley (1991): Physiological characteristics of elite judo athletes. Int J Sports Med 12(2):196-203.

7- Casals, Cristina, Jesús Huertas, Emerson Franchini, Katarzyna SterkowiczPrzybycień, Stanisław Sterkowicz, Carlos Gutiérrez-García, and Raquel EscobarMolina (2017): Special Judo Fitness Test Level and Anthropometric Profile of Elite Spanish Judo Athletes. Journal of strength and conditioning research / National Strength \& Conditioning Association 31:1229-35.

8- Franchini, E., F. B. Del Vecchio, K. A. Matsushigue, and G. G. Artioli (2011a): Physiological profiles of elite judo athletes. Sports Med 41(2):147-66.

9- Franchini, E., S. Sterkowicz, U. Szmatlan-Gabrys, T. Gabrys, and M. Garnys. (2011b): Energy system contributions to the special judo fitness test. Int J Sports Physiol Perform 6(3):334-43.

10- Franchini, Emerson, Fabrício Abcde, Fabricio Del Vecchio, Vecchio, Stanislaw Cde, Stanisław Sterkowicz, and Acde (2009): A special judo fitness test classificatory table. Archives of Budo 5:127-29.

11- Franchini, Emerson, Fabricio Del Vecchio, Karin Matsushigue, and Guilherme Artioli (2011c): Physiological Profiles of Elite Judo Athletes. Sports medicine 
(Auckland, N.Z.) 41:147-66.

12- Franchini, Emerson, U. F. Julio, Valéria Panissa, Fabio Lira, Marcus Agostinho, and Braulio Branco (2017): Short-term low-volume high-intensity intermittent training improves judo-specific performance. Archives of Budo 12:219-29.

13- Franchini, Emerson, Fabio Nakamura, Monica Takito, Marian Kiss, and Stanisław Sterkowicz (1998): Specific fitness test developed in Brazilian judoists. Biology of Sport 15:165-70.

14- Franchini, Emerson, Alexandre Nunes, Josué Moraes, and Fabricio Del Vecchio (2007): Physical Fitness and Anthropometrical Profile of the Brazilian Male Judo Team. Journal of physiological anthropology 26:59-67.

15- Franchini, Emerson, Juliano Schwartz, and Monica Yuri Takito (2018): Maximal isometric handgrip strength: comparison between weight categories and classificatory table for adult judo athletes. J Exerc Rehabil 14(6):968-73.

16- Franchini, Emerson, Juliano Schwartz, and Monica Yuri Takito (2020): Maximal isometric handgrip strength in judo athletes from different age groups. Sport Sciences for Health 16(1):93-98.

17- Franchini, Emerson, Monica Takito, and Rômulo Bertuzzi (2005): Morphological, physiological and technical variables in high-level college judoists. Archives of Budo $1: 1-7$.

18- Fukuda, David, Jeffrey Stout, Patrick Burris, and Robert Fukuda (2011): Judo for Children and Adolescents: Benefits of Combat Sports. Strength \& Conditioning Journal 33:60-63.

19- Kamimura, T., and Y. Ikuta (2001): Evaluation of grip strength with a sustained maximal isometric contraction for 6 and 10 seconds. J Rehabil Med 33(5):225-29.

20- Katralli, Jayasudha, and Shivaprasad S. Goudar (2012): Anthropometric Profile and Special Judo Fitness levels of Indian Judo Players. Asian journal of sports medicine 3(2):113-18.

21- Kons, Rafael Lima, Juliano Dal Pupo, Jonathan Ache-Dias, Thyago Garcia, Romário Rodrigues da Silva, Luiz Felipe Guarise Katicips, and Daniele Detanico (2018): Effect of official judo matches on handgrip strength and perceptual responses. J Exerc Rehabil 14(1):93-99.

22- Lopes-Silva, João, Valéria Panissa, Ursula Julio, and Emerson Franchini (2018): Influence of Physical Fitness on Special Judo Fitness Test Performance - A Multiple Linear Regression Analysis. The Journal of Strength and Conditioning Research. doi:10.1519/JSC.0000000000002948.

23- Marques, V., V. Coswig, R. Viana, A. Leal, F. Alves, A. Alves, G. Teles, C. Vieira, M. Silva, D. Santos, and P. Gentil (2019): Physical Fitness and Anthropometric Measures of Young Brazilian Judo and Wrestling Athletes and Its Relations to Cardiorespiratory Fitness. Sports (Basel) 7(2).

24- Murata, Hiroko, Satomi Oshima, Suguru Torii, Motoko Taguchi, and Mitsuru Higuchi (2016): Characteristics of body composition and cardiometabolic risk of Japanese male heavyweight Judo athletes. Journal of physiological anthropology $35(1): 10$.

25- Nikolova, Anna, Diana Dimitrova, and Lubomir Mladenov (2020): AEROBIC CAPACITY OF JUDO PLAYERS SCALED FOR DIFFERENCES IN BODY SIZE. Journal of Applied Sports Sciences 2.

26- Sertić, Hrvoje, Stanisław Sterkowicz, and Dinko Vuleta (2009): Influence of latent motor abilities on performance in judo. Kinesiology 41:76-87.

27- Sterkowicz-Przybycień, Katarzyna, and David Fukuda (2014): Establishing 
Normative Data for the Special Judo Fitness Test in Female Athletes Using Systematic Review and Meta-Analysis. Journal of strength and conditioning research / National Strength \& Conditioning Association 28.

28- Sterkowicz-Przybycień, Katarzyna, Bianca Miarka, and David Fukuda (2016): Sex and Weight Category Differences in Time-Motion Analysis of Elite Judo Athletes: Implications for Assessment and Training. Journal of Strength and Conditioning Research 31:1.

29- Tomlin, D. L., and H. A. Wenger (2002): The relationships between aerobic fitness, power maintenance and oxygen consumption during intense intermittent exercise. J Sci Med Sport 5(3):194-203.

30- Torres-Luque, Gema, Hernández-García Raquel, Raquel Escobar-Molina, Nuria Garatachea, and Pantelis Nikolaidis (2016): Physical and Physiological Characteristics of Judo Athletes: An Update. Sports 4:20.

31- Trossman, Patricia B., and Ping-Wu Li (1989): The Effect of the Duration of Intertrial Rest Periods on Isometric Grip Strength Performance in Young Adults. The Occupational Therapy Journal of Research 9(6):362-78. 